1. Member, Work Group, Minimum Technical Standards And Recommendations For Rehabilitation For Emergency Medical Teams' Guidance Document, World Health Organization, Geneva/Switzerland

2. Lead, Work Group, 'minimum Technical Standards And Recommendations For Rehabilitation For Emergency Medical Teams' Guidance Document, World Health Organization, Geneva/Switzerland

Study/Objective: To disseminate the development and use to date, of the WHO 'Minimum Technical Standards and Recommendations for Rehabilitation for Emergency Medical Teams' guidance document (e-publication expected December 2016).

Background: The World Health Organization (WHO) Emergency Medical Team (EMT) initiative, supports populations severely impacted by large-scale catastrophic disasters by ensuring a rapid, professional, coordinated medical response by national and international teams. Physical rehabilitation has become increasingly recognized as an essential health component of the medical response in disasters, due to the humanitarian imperative to limit long-term disability, and optimize functional outcomes in persons sustaining severe traumatic injuries (and infectious disease outbreak sequelae). The WHO 'Minimum Technical Standards and Recommendations for Rehabilitation for Emergency Medical Teams' was developed to provide guidance for integrating rehabilitation capacity into EMTs, and hence the global humanitarian medical response.

Methods: Guidance document development was a highly consultative process hosted by WHO involving global experts from the rehabilitation field, including operational rehabilitation from International Non-Governmental Organizations (INGOs), international professional rehabilitation societies, and individuals. Results: The WHO 'Minimum Technical Standards and Recommendations for Rehabilitation for Emergency Medical Teams' guidance document draft, is available on the WHO EMT Initiative extranet, with the official e-publication anticipated in December 2016. The minimal technical standards for rehabilitation have been incorporated into the verification process undergone by EMTs, to qualify for global classification. It is expected that use of the minimum standards and recommendations will result in expanded, quicker access of patients to rehabilitation services (and equipment) in disasters, as well as improved referrals between EMTs and local health facilities for ongoing rehabilitation service provision - translating to increased near-term functional outcomes and reduced long-term disability for affected persons.

Conclusion: In conclusion, the WHO 'Minimum Technical Standards and Recommendations for Rehabilitation for Emergency Medical Teams' guidance document establishes minimum standards for rehabilitation to increase the rehabilitation capacity of EMTs in disasters (and outbreaks).

Prehosp Disaster Med 2017;32(Suppl. 1):s91-s92

doi:10.1017/S1049023X17002370

\section{International Emergency Medical Teams in the Russian}

\section{Federation}

Gennady Kipor ${ }^{1}$, Sergei Goncharov ${ }^{2}$, Valeri Shabanov ${ }^{3}$, Michel Bistrov ${ }^{2}$, Natalia Pichugina 4 , N. Baranowa ${ }^{5}$

1. International, All Russian Center for Disaster Medicine "Zaschta", Moscow/Russian Federation
2. Directorat, All Russian Center for disaster Medicine "Zaschita", Moscow/Russian Federation

3. Mobile Field Hospital, All Russian Center for Disaster Medicine "Zaschta", Moscow/Russian Federation

4. Administration, All Russian Center for disaster Medicine "Zaschita", Moscow/Russian Federation

5. Chief Of Medical Evacuation Team, All Russian Center for disaster Medicine "Zaschita”, Moscow/Russian Federation

Study/Objective: To show the tools and tasks of Emergency medical teams in the Russian Federation.

Background: International experience of a national Mobile Field Hospital in the Russian Federation (RF) is very large (Columbia, Turkey, Iran, China, Afghanistan, Chile, etc). The Task of this presentation is not to show the procedures of WHO in step-by-step certification of International Emergency Medical Teams, but rather how this processing was created in the Russian Federation.

Methods: Procedure analysis.

Results: 1. In Civil Law Code there is a special legislative article, supported by some part of the state budget, for humanitarians, free of charge assistance delivery for the injured in emergencies anywhere. 2. Medical emergency relief in all aspects is never connected to policy, politics, confession, economical status of patient, etc. Protocols and Standards are the same to everyone injured independently upon his social position. 3. Every central hospital or specialized clinic has bed reserves (5\%) if any emergency occurs. 4. All the system of Emergency Medical Care in RF has its satellite network, and taking it into account, could connect all the medical facilities and register of specialists into one competent and powerful telemedicine framework. 5. The system has mobile field hospitals, portable modern equipment, and staff prepared who are regularly educated and trained. 6 . The system is strictly organized, centralized vertically, and is under the management of the RF Health Ministry, 7. The system is strictly territorially organized and has more than 80 territorial and regional units (centers). In such a way, all Russian Disaster Medicine Centers 'Zaschita' (Protection) have become one of the first in the great number of medical facilities amidst many WHO member-countries who satisfies the requirements of WHO procedures of certification.

Conclusion: Tasks of the internal emergency medical teams of RF, Disaster Medicine Centre 'Zaschita', as a collaborating WHO disaster medicine center, are presented and discussed.

Prehosp Disaster Med 2017;32(Suppl. 1):s92

doi:10.1017/S1049023X17002382

Learning from Canadian Red Cross International Health

Team Deployments: Understanding Individual and

Institutional Competencies that would be Beneficial

to a Canadian Domestic Response

Lynda Redwood-Campbell ${ }^{1}$, Salim Sohani ${ }^{2}$

1. Family Medicine, McMaster University, Hamilton/Canada

2. International Operations, Canadian Red Cross, Ottawa/ON/Canada

Study/Objective: To determine the types of individual/ institutional competencies/skills that are obtained from working with the Canadian Red Cross (CRC) International 